\title{
Post-Newtonian simulations of super-massive black hole binaries in galactic nuclei
}

\author{
I. Berentzen ${ }^{1, \star}$, M. Preto ${ }^{1}$, P. Berczik ${ }^{1,2}$, D. Merritt $^{3}$, and R. Spurzem ${ }^{1}$ \\ 1 Astronomisches Rechen-Institut, Zentrum für Astronomie, Universität Heidelberg, Mönchhofstr. 12-14, D-69120 Hei- \\ delberg, Germany \\ 2 Main Astronomical Observatory, National Academy of Sciences of Ukraine, 27 Akademika Zabolotnoho St., 03680 \\ Kyiv, Ukraine \\ ${ }^{3}$ Center for Computational Relativity and Gravitation, Rochester Institute of Technology, 78 Lomb Memorial Drive, \\ Rochester, NY 14623
}

The dates of receipt and acceptance should be inserted later

Key words galaxies: nuclei - methods: $N$-body simulations - black hole physics - gravitational waves

We present some preliminary results from recent numerical simulations that model the evolution of super-massive black hole (SMBH) binaries in galactic nuclei. Including the post-Newtonian terms for the binary system and adopting appropriate models for the galaxies allows us, for the first time, to follow the evolution of SMBH binaries from kpc scales down to the coalescence phase. We use our results to make predictions of the detectability of such events with the gravitational wave detector LISA.

(c) 2008 WILEY-VCH Verlag GmbH \& Co. KGaA, Weinheim

\section{Introduction}

The formation and evolution of super-massive black holes (SMBHs) in galactic nuclei is an important ingredient for our understanding of galaxy formation and evolution. In the hierarchical cosmological framework, galaxies merge in the course of their evolution. Therefore, either many massive binary (or even multiple) SMBHs should exist, or else we need to develop a detailed understanding of what ultimately happens to the massive binaries. According to the standard theory, the evolution of SMBHs after a galaxy merger can be divided in three stages (Begelman, Blandford \& Rees 1980): (i) Dynamical friction causes the individual SMBHs to spiral to the galactic centre where they form a binary. (ii) Super-elastic scattering of stars causes the binary to harden. (iii) Eventually, the binary may coalesce owing to the emission of gravitational waves. However, this is only possible if stellar- and gas-dynamical processes have first brought the two SMBHs to small enough separations (some $10^{-3} \mathrm{pc}$ ) that the emission of gravitational waves is significant. Whether Nature typically succeeds in overcoming this "final parsec problem," (see review by Milosavljević \& Merritt 2003) is currently unknown.

Here we present preliminary results of recent $N$-body simulations of SMBH binaries in galactic nuclei, including post-Newtonian $(\mathcal{P N})$ equations of motion up to the $2.5 \mathcal{P N}$ order. In Sec. 2 we describe our $\mathcal{P N}$ implementation and provide numerical tests and simple two-body experiments. In Sec. 3 we summarise some first results of our detailed galactic nuclei simulations, following the evolution of the

\footnotetext{
^ Corresponding author: e-mail: iberent@ari.uni-heidelberg.de
}

SMBH binaries from the unbound state down to relativistic coalescence. We conclude with Sec.4.

\section{A two-body Hermite $\mathcal{P N}$ integrator}

In order to incorporate the dynamics of relativistic binary systems into our direct $N$-body code (see Sec. 3) we use the $\mathcal{P N}$ equations of motion, which are expressed by expanding the relativistic acceleration between two compact objects in a power series of $1 / c$ (e.g., Damour \& Deruelle 1981; Soffel 1989). Schematically, the $\mathcal{P N}$ equation of motion for an object in the binary can be written as:

$$
\mathbf{a}=\mathbf{a}_{0}+\frac{1}{c^{2}} \mathbf{a}_{1}+\frac{1}{c^{4}} \mathbf{a}_{2}+\frac{1}{c^{5}} \mathbf{a}_{2.5}+\mathcal{O}\left(\frac{1}{c^{6}}\right),
$$

where $\mathbf{a}_{0}$ is the classical Newtonian acceleration, $\mathbf{a}_{1}$ and $\mathbf{a}_{2}$ are the energy conserving $1 \mathcal{P N}$ and $2 \mathcal{P N}$ corrections, respectively, and $\mathbf{a}_{2.5}$ is the dissipative $2.5 \mathcal{P N}$ term, describing the dominant damping forces due to gravitational wave emission. In the current $\mathcal{P N}$ routine we neglect any effects of spin-orbit and spin-spin coupling (e.g., Damour 1987). For the full expressions of the individual terms up to $3.5 \mathcal{P N}$ order see, e.g., Blanchet (2006). We implement the equations of motion formulated in a general harmonic (Cartesian) coordinate system, whereas other astrophysical implementations often use expressions given in the binary's centre of mass frame (e.g., Kupi, Amaro-Seoane \& Spurzem 2006; Aarseth 2007).

In order to achieve high accuracy in the simulations we use a $4^{\text {th }}$ order Hermite integrator (e.g., Makino \& Aarseth 1992), which requires in addition to the accelerations also 


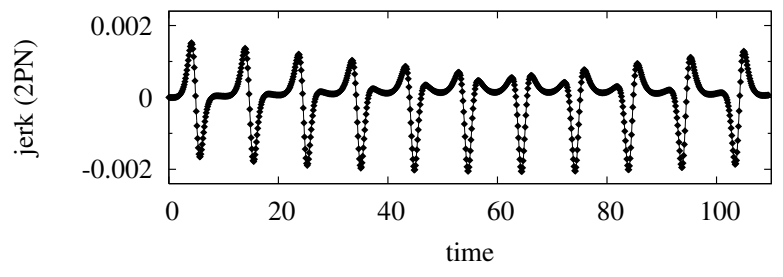

Fig. 1 Comparison of the $\mathcal{P N}$ implementation (solid line) and the finite differences $\Delta a_{i} / \Delta t_{i}$ (symbols). Shown are the curves for the $2 \mathcal{P N} \mathrm{x}$-component of some (arbitrary) orbit integration.

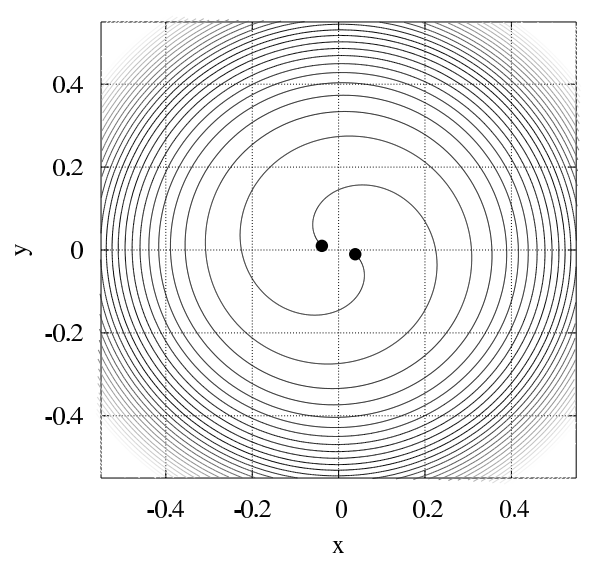

Fig. 2 Inspiral and plunge of an equal-mass binary system using the post-Newtonian equations of motion. The numerical integration is done using a $4^{\text {th }}$ order Hermite integrator and hierarchical time-step scheme.

their first time-derivatives ("jerk") - including the $\mathcal{P N}$ corrections. Since the $\mathcal{P N}$ accelerations and jerks are complicated functions of the particles' masses, positions and velocities, their derivation and numerical implementation is a potential source of error. Therefore, we provide some numerical tests which turned out to be useful benchmarks, as already applied with other $\mathcal{P N}$ implementations (e.g., Kupi et al. 2006; Löckmann \& Baumgardt 2008):

(i) Time-derivatives. To check the correct implementation of the jerks of the $\mathcal{P N}$ terms, we evolve a binary system for several orbital periods and compare the finite differences $\Delta \mathbf{a}_{\mathcal{P N}} / \Delta t$ for consecutive time-steps to the directly calculated a from our implementation (Fig. 1). Any deviations between the two would be easily detectable and clearly indicate any errors in the values of the computed jerk. In Fig. 2 we show a typical trajectory of an binary inspiral integrated with our $\mathcal{P N}$ code, starting with a quasi-circular orbit.

(ii) Merging time for eccentric binary orbits. In the following test we set the gravitational constant $G$ and the two particle masses to unity. The speed of light in our model units is set to $c=101$ The two particles are placed on the

1 These units are chosen for numerical testing purposes only and are not physically motivated.
Table 1 The columns from left to right give for each integrated orbit the corresponding $\left(1-e_{0}^{2}\right)$, the initial eccentricity $e_{0}$, the initial semi-major axis $a_{0}$, and the two 'merging' times $T_{\mathcal{P N}}$ and $T_{2.5 \mathcal{P N}}$, respectively.

\begin{tabular}{cccrr}
\hline $1-e_{0}^{2}$ & $e_{0}$ & $a_{0}$ & $\mathrm{~T}_{\mathcal{P N}}$ & $\mathrm{T}_{2.5 \mathcal{P N}}$ \\
\hline 1.000 & 0.000 & 2.000 & 16666.2 & 15623.4 \\
0.950 & 0.224 & 1.635 & 5741.4 & 5783.1 \\
0.900 & 0.316 & 1.519 & 3370.4 & 3554.0 \\
0.850 & 0.387 & 1.442 & 2125.9 & 2347.1 \\
0.800 & 0.447 & 1.382 & 1377.7 & 1598.0 \\
0.750 & 0.500 & 1.333 & 899.7 & 1102.7 \\
0.700 & 0.548 & 1.292 & 585.3 & 763.7 \\
0.650 & 0.592 & 1.257 & 365.6 & 527.1 \\
0.600 & 0.632 & 1.225 & 234.6 & 360.5 \\
0.550 & 0.671 & 1.197 & 141.7 & 242.9 \\
0.500 & 0.707 & 1.172 & 80.3 & 159.7 \\
\hline
\end{tabular}

$x$-axis with an initial separation of $\Delta x=2$. The initial velocities $\pm v_{y}$ are calculated for a Keplerian orbit of given eccentricity $e_{0}$. We then integrate the orbits using (a) the full $\mathcal{P N}$ (i.e., in our case up to $2.5 \mathcal{P N}$ ) corrections and (b) applying only the $2.5 \mathcal{P N}$ correction. In both cases the integrations are stopped at the times $T_{\mathcal{P N}}$ and $T_{2.5 \mathcal{P N}}$, respectively, when the binaries reach a separation of 10 Schwarzschild radii $\left(R_{\mathrm{S}}=2 G m_{\mathrm{bh}} / c^{2}\right)$, i.e., well before the $\mathcal{P N}$ approximation may become inadequate. The results for different initial eccentricities are given in Table 1 The numbers given in Table 1 are in agreement with integrations using the $\mathcal{P N}$ implementations of Kupi et al. (2006) and Aarseth (2007). The exact result may slightly vary for different integration schemes and time-step controls.

In Fig. 3] we show a comparison of the "merging" times $T_{\mathcal{P N}}$ (solid line) and $T_{2.5 \mathcal{P N}}$ (dashed line). Note that the results can vary by a factor of a few with increasing eccentricity. This underlines the importance of the lower order $\mathcal{P N}$ corrections, which sometimes have been neglected in earlier astrophysical simulations. The pericentre shift due to the $1 \mathcal{P N}$ and $2 \mathcal{P N}$ terms leads to more pericentre passages and therefore to an overall stronger dissipation (especially for high eccentricities), which shortens $T_{\mathcal{P N}}$ as compared to $T_{2.5 \mathcal{P N}}$. The almost horizontal part of the two curves in Fig. [3 $\left(1-e_{0}^{2} \approx 0.2 \ldots 0.4\right)$ is the result of a direct plunge into the $10 R_{\mathrm{S}}$ region due to the high eccentricity and small pericentre distances. Finally, we compare our results to the numerical integration of the equations given by Peters (1964), describing the orbit-averaged changes of the eccentricity and semi-major axis due to gravitational wave emission. We find good agreement between the latter integration and our corresponding $2.5 \mathcal{P N}$ two-body integrations. The diverging behaviour for $\left(1-e_{0}^{2}\right)<0.4$ comes from the fact that our orbit-averaged integration is terminated when the semimajor axis (instead of the binary separation) reaches $10 R_{\mathrm{S}}$.

(iii) The innermost stable circular orbit (ISCO). Here we study the linear stability of circular orbits. It is known for binary systems that circular orbits with radii below a certain radius $r_{\mathrm{I}}$ are unstable, even without dissipative ef- 


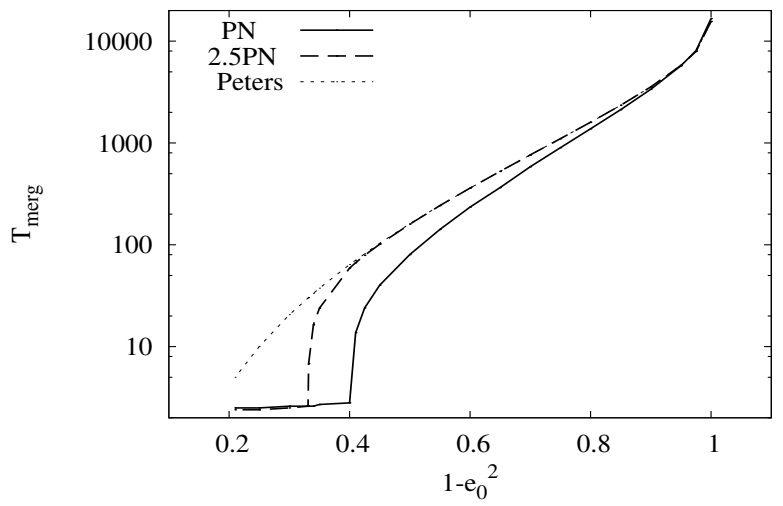

Fig. 3 Shown are the results obtained from numerical integration of the orbit-averaged equations from Peters (1964) (dotted line) and from the direct two-body integration including the $2.5 \mathcal{P N}$ correction (dashed line) and full $\mathcal{P N}$ corrections.

fects. The evolution of an relativistic binary changes from a quasi-adiabatic inspiral to an unstable plunge when crossing $r_{\text {I }}$ (e.g., Kidder, Will \& Wiseman 1993). Analytically, the radius $r_{\mathrm{I}}$ of the ISCO can be calculated using the standard linear stability analysis (e.g., Blanchet \& Iyer 2003). To determine $r_{\text {I }}$ on an ISCO for objects of comparable mass we use here the exact $2 \mathcal{P N}$ accurate circular frequencies $\omega_{2 \mathcal{P N}}$ as given in Preto (2007). We find for the binary system described in test (ii) that the ISCO has a radius of $r_{\mathrm{I}} \sim 0.136$ in our model units (Preto 2007). Now we determine $r_{\text {I }}$ by numerical integration of circular $\mathcal{P N}$ orbits. The model units and masses are the same as the ones used in test (ii). The initial velocities of the two particles are calculated based on the $2 \mathcal{P N}$ accurate $\omega_{2 \mathcal{P N}}$ (see above). We integrate the $\mathcal{P N}$ circular orbits including only $1 \mathcal{P N}$ and $2 \mathcal{P N}$ corrections, i.e., under the absence of gravitational wave emission. The integration of the orbits has been terminated at a time $t_{\text {term }}$ either after an unstable plunge or after a total maximum of $n_{i}$ (initial) orbital periods $T_{\text {orb }}$. In Fig. 4 we show $t_{\text {term }} /\left(n_{i} \times T_{\text {orb }}\right)$ for $n_{i}=100$ (solid line), $n_{i}=500$ (dotted line) and $n_{i}=1000$ (dashed line). As an indicator for stability we also plot the final ratio $r_{\text {term }} / r_{0}$, where $r_{\text {term }}$ is the separation of the particles at time $t_{\text {term }}$. We find that our $\mathcal{P N}$ two-body integration accurately captures the radius of the ISCO in all three cases. The transition from the stable to the unstable regime is very abrupt as the theory predicts, and happens at the radius estimated by the linear stability analysis.

\section{Super-massive black hole binaries in galactic nuclei}

In this section we present preliminary results of recent direct $N$-body simulations of SMBH binaries in galactic nuclei including $\mathcal{P N}$ corrections (Berentzen et al. 2008). Initial con-

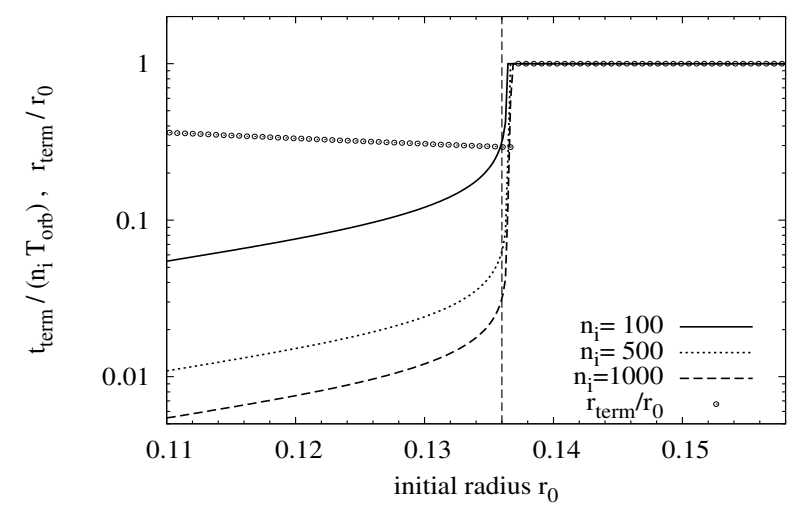

Fig. 4 Normalized integration time $t_{\text {term }} /\left(n_{i} \times T_{\text {orb }}\right)$ for initially circular orbits with radius $r_{0}$. We show our results for $n_{i}=100$ (solid line), $n_{i}=500$ (dotted line) and $n_{i}=1000$ (dashed line). The symbols show the final ratio $r_{\text {term }} / r_{0}$. The vertical line indicates the radius of the innermost stable circular orbit (ISCO) as derived from the linear stability analysis. Note that our $\mathcal{P N}$ code is capable of accurately capturing the ISCO.

ditions were based on the models of Berczik et al. (2006). The initial field particle distribution was a discrete realisation of the axisymmetric, rotating King (1966) models of Longaretti \& Lagoute (1996). We consider such models to be reasonable representations of galactic nuclei in post-merger galaxies. When the rotation parameter is sufficiently large, the models evolve rapidly into triaxial bars. Berczik et al. (2006) found that in this case, the massive binary continued to harden at an essentially $N$-independent rate, due presumably to the centrophilic nature of the orbits (Merritt \& Poon 2004). This feature means that evolution of the massive binary can be faithfully reproduced even with modest particle numbers. Accordingly, the nucleus was represented here using either $N=25 \times 10^{3}$ or $N=50 \times 10^{3}$ particles, in each case with nine different random realisations. The masses of the two SMBH particles were chosen to be one percent of the total mass. For the simulations we used an updated version of the publically available $\varphi$-GRAPE code (Harfst et al. 2007). The code is an NBODY1-like algorithm (Aarseth 1999), including a hierarchical time-step scheme and a $4^{\text {th }}$-order Hermite integrator. Our modified version includes the $\mathcal{P N}$ implementation described in the previous section. Our simulations were carried out on the high-performance GRAPE6A clusters at the Astronomisches Rechen-Institut (Heidelberg), Rochester Institute of Technology (New York) and the Main Astron. Observatory (Kiev). More details on the code and hardware are given in Berentzen et al. (2008) and Spurzem et al. (2008).

In Fig. 5 we show the evolution of the inverse semimajor axis $1 / a$ and eccentricity $e$ of the binaries in our

${ }^{2}$ http://wiki.cs.rit.edu/view/GRAPEcluster/phiGRAPE 


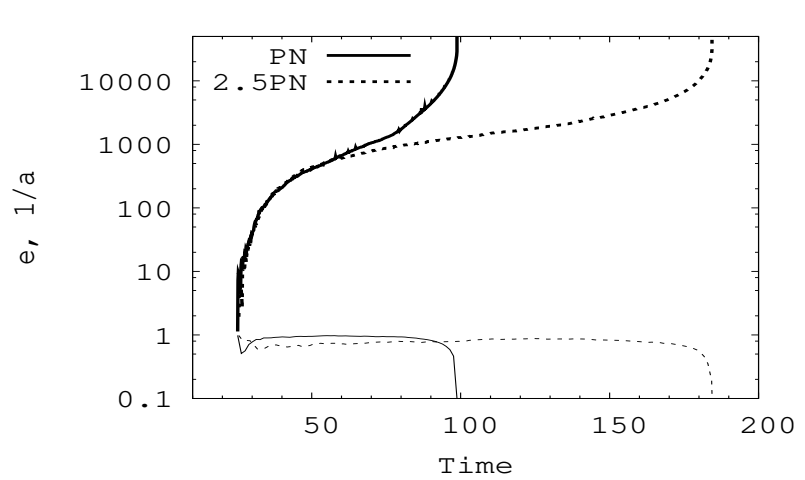

Fig. 5 Evolution of the SMBH binaries in a model of a rotating galactic nucleus. Shown are the orbital eccentricity $e$ (thin lines) and the inverse semi-major axis (thick lines) as a function of time. The full and dashed lines represent models using the full $\mathcal{P N}$ and $2.5 \mathcal{P N}$ corrections, respectively. Note the significantly different evolution of the binary.

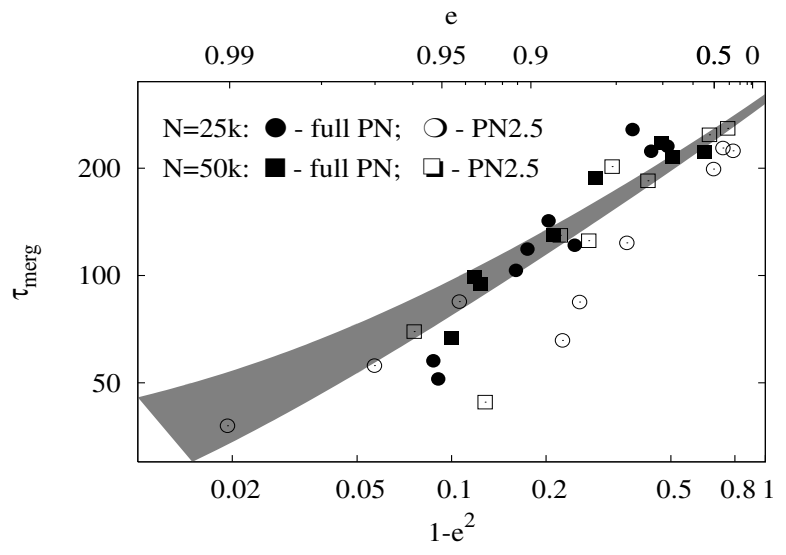

Fig. 6 Merging time $\tau_{\text {merg }}$ as a function of eccentricity $e$. Shown are simulations with $25 \mathrm{k}$ (circles) and 50k (squares) particles. The open and filled symbols represent models with full $\mathcal{P N}$ and only $2.5 \mathcal{P N}$ corrections, respectively. The gray region marks the theoretically estimated merging times (see Berentzen et al. 2008).

simulations with full $\mathcal{P N}$ (solid lines) and with only $2.5 \mathcal{P N}$ corrections (dashed lines). Initially the two SMBH particles are unbound; they form a very eccentric binary at $t \sim 25$. Afterwards the binaries steadily harden mainly by stellardynamical processes, before relativistic effects start to dominate their evolution. Eventually, they reach the inspiral phase during which the orbits circularise before the final coalescence. As expected, we find that the lower order $\mathcal{P N}$ terms significantly influence the evolution of the binaries and must not be neglected in this type of simulation. Finally, we show in Fig. 6 the merging times $\tau_{\text {merg }}$ for our SMBH binaries as a function of $e$. Scaling our model units to real galaxies we find that $\tau_{\text {merg }}$ in our models is typically less than $1 \mathrm{Gyr}$ after the binary has formed.

\section{Summary}

We upgraded the direct $N$-body code $\varphi$-GRAPE by including a relativistic treatment for binary SMBHs via the $\mathcal{P N}$ equations of motion up to $2.5 \mathcal{P N}$ order. The new implementation was carefully tested for accuracy. We then carried out numerical simulations of SMBH binaries in galactic nuclei. Our models allow us, for the first time, to follow the evolution of initially unbound SMBHs with kiloparsec-scale separations down to relativistic inspiral and coalescence. The SMBH binaries in our simulations form generally with high eccentricities, which are maintained during the evolutionary phase dominated by stellar-dynamical effects. We have shown that the inclusion of the lower order $1 \mathcal{P N}$ and $2 \mathcal{P N}$ corrections is crucial for obtaining the correct time dependence of the binary orbital parameters and final coalescence time. Our results demonstrate that $\mathrm{SMBH}$ binaries in certain models of galactic nuclei can overcome the stalling barrier by stellar-dynamical effects alone. A detailed description and discussion of our models and results will be given in Berentzen et al. (2008) and in Preto et al. (2008).

Acknowledgements. We cordially would like to thank S. Aarseth, P. Amaro-Seoane, A. Gopakumar, A. Gualandris, S. Harfst, G. Kupi and G. Schäfer for valuable suggestions and fruitful discussions. This work was supported by Volkswagen Foundation (GRACE, Ref. I/80 041-043), DLR (Deutsches Zentrum für Luft- und Raumfahrt), and SFB 439 of DFG. I.B. thanks the conference organisers for financial support.

\section{References}

Aarseth, S. J.: 1999, PASP 111, 1333

Aarseth, S. J.: 2007, MNRAS 378, 285

Begelman M. C., Blandford R. D., Rees M. J.: 1980, Nature 287, 307

Berczik, P., Merritt, D., Spurzem, R., Bischoff, H.-P.: 2006, ApJ 642, L21

Berentzen, I., Preto, M., Berczik, P., Merritt, D., Spurzem, R.: 2008, to be subm.

Blanchet, L.: 2006, LLR 9, 4. URL ${ }^{3}$

Blanchet, L., Iyer, B. R.: 2003, CQGRa 20, 755

Damour, T.: 1987, in: S. W. Hawking and W. Israel (eds.), 300 Years of Gravitation, Cambridge Univ. Press, p. 128

Damour, T., Deruelle, N.: 1981, PhyL. 87, 81

Harfst, S., Gualandris, A., Merritt, D., Spurzem, R., Portegies Zwart, S., Berczik, P.: 2007, NewA. 12, 357

Kidder, L. E., Will, C. M., Wiseman A. G.: 1993, PhRvD 47, 3281

King, I. R.: 1966, AJ 71, 276

Kupi, G., Amaro-Seoane, P., Spurzem, R.: 2006, MNRAS 371, L45

Löckmann, U., Baumgardt, H.: 2008, MNRAS 384, L323

Longaretti, P.-Y., Lagoute, C.: 1996, A\&A 308, 453

Makino, J., Aarseth, S. J.: 1992, PASJ 44, 141

Merritt, D., Poon, M. Y.: 2004, ApJ 606, 788

Milosavljević, M., Merritt, D.: 2003, in: The Astrophysics of Gravitational Wave Sources, AIPC 686, p. 201

Peters, P.C.: 1964, PhRvB 136, 1224

Preto, M.: 2007, Ph.D. thesis, University of Heidelberg

\footnotetext{
${ }^{3}$ http://www.livingreviews.org//rr-2006-4
} 
Preto, M., Berentzen, I., Berczik, P., Merritt, D., Spurzem, R.: 2008 , in prep.

Soffel, M. H.: 1989, in: Relativity in Astrometry, Celestial Mechanics and Geodesy, XIV, 208, p. 32

Spurzem, R., Berentzen, I., Berczik. P., Merritt, D., AmaroSeoane, P., Harfst, S., Gualandris, A.: 2008, in: S.J. Aarseth, C.A. Tout \& R.A. Mardling (eds.), The Cambridge $N$-body Lectures. LNP. (in press) 